\title{
Bilingual pronunciation and first language dominancy
}

\author{
Rohini Chandrica Widyalankara \\ English Language Teaching Unit University of Kelaniya Sri Lanka
}

\begin{abstract}
Literature on Sri Lankan English, through reasonable deduction, states that deviations from Standard Sri Lankan English (SSLE) pronunciationis a feature of Sinhala dominancy in Sinhala/Other Variety Sri Lankan English (S/OVSLE) bilinguals or Tamil dominancy in Tamil/Other Variety Sri Lankan English (T/OVSLE) bilinguals. This study using standard statistical procedures collates the difference in the mean values of self-declared competence in First Language (L1) and Second Language (L2) across the four skills: Reading, Writing, Speaking, and Listening with the difference in rate of usage of L1 and L2 in selected social and personal domains to calculate L1 dominancy in each bilingual participant $(n=185)$. Then the rate of occurrence of selected deviations from SSLE pronunciation in each participant is estimated. The correlation between the independent variable L1 dominancyand the dependent variable rate of occurrence of selected deviations from SSLE pronunciation in the populations is illustrated through scatter diagrams, calculation of the Pearson Correlation and the Coefficient of Determination. Statistical analysis shows that there is a medium $9 \%$ and a moderate $11 \%$ correlation between L1 dominancy and rate of occurrence of deviations from SSLE pronunciation in S/OVSLE and T/OVSLE bilinguals respectively.
\end{abstract}

Keywords: Sri Lankan English pronunciation, bilingualism, L1 dominancy, self-declared competence, language selection in functional domains, Willingness to Communicate

\section{Heuristics In Bilingual Taxonomy And Language Dominancy}

Defining bilingualism this study identifies a plethora of definitions ranging from the restrictive to the very flexible. A multitude of interdisciplinary theory and experimental research bear evidence for the complex nature and the increasing ambit of bilingualism on socio- psycho - neuro linguistics. Bilingualism has been branded as a 'ubiquitous yet an abstruse word, and perhaps used rather esoterically in different fields of linguistics' (Sia\&Dawaele, 2006). The general state of flux within the domain of bilingualism is reflected through the polemics in literature where the traditional definitions seem incongruous in the sphere of modern linguistics. Literature reveals that early definitions on bilingualism were narrow and prescriptive and focused on 'native like control on two languages' (Bloomfield, 1935: 56) where equal fluency in both languages was a marker of bilingualism. Opposition comes from Grosjean (1997) who refutes the misconception that bilinguals are 'equally fluent in their languages' and further states that 'a bilingual is not simply the sum of two monolinguals'. Hakuta (1986) too identifies the difficulty in defining 'native like control'.

Haugen (1953: 7) provided a minimalist definition based on the onset of bilingualism. According to Haugen bilingualism begins at a point where the speaker of one language can produce 'complete meaningful utterances in the other language'. But evidence of minimal function in a language reflected through 'complete meaningful utterances' such as reeling off the words of a popular English song, according to Beardsmore (1982), does not provide sufficient proof for beginnings of bilingualism. An example to supplement the argument comes from the Sri Lankan English (SLE) usages 'see you later', 'no problem' which might be habitually used by interlocutors during Sinhala discourse who would find the construction of a standard 'complete meaningful utterance' in English beyond their linguistic capabilities.

Other definitions are deliberately made vague. 'It seems obvious that if we are to study the phenomenon of bilingualism we are forced to consider it as something entirely relative' (Mackey, 1957: 51). An equally vague definition for bilingualism comes from another proponent of relativism in bilingualism. According to Arsenian(1937: 19) 'One of the most important factors to be borne in mind when discussing bilingualism of any type is that a notion of relativism must be introduced where by the degree of bilingualism under analysis has to be ascertained'. But according to Beardsmore (1982: 7) 'the degree of bilingualism is not an easy thing to measure'. Crystal (2003: 51) too identifies 'many kinds and degrees of bilingualism'.

In essence, distancing from narrow, prescriptive, historical definitions this study bears acquiescence to the following: 'bilingualism must be able to account for the presence of two languages within one and the same speaker, remembering that ability in these two languages may or may not be equal' (Beardsmore 1982: 3) and recognize that 'bilinguals have conceptual representations linked to two different lexical representations; (Abutalebi\& Green, 2007; de Groot, 2010; Finkbeiner et al., 2006; Francis, 1999; Kroll \& Stewart, 1994). Thus the ontology within the domain of bilingualism identifies the elements equilingual/balanced bilingual, and 
bilinguals with a dominant and a weaker language within the bilingual's code repertoire among other classifications. They often are broad terms of reference which attempt to provide heuristic taxonomies devoid of justification and validation.

The typology relevant to this study identifies the element language dominancy. According to Beardsmore (1982:7 ${ }^{[17]}$ Equilingualism (alternately called balanced bilingualism) occurs when a speaker's mastery of two languages is roughly equivalent and the individual has the ability to function equally well in two languages within a given society'. He further states that this ability is 'clearly discernible from two monoglot speakers of the respective languages through possible traces of phonological, morphological or syntactic interference in both'.Given that the competence of the two languages may vary according to functional specialization, Beardsmore (ibid: 31$)^{[18]}$ states 'it is wiser to talk about a bilingual's dominant language'.

\section{Sociolinguistic Background}

Contact linguistic dynamics between the historical input variety British English (BE)and the two main vernaculars Sri Lanka: Sinhala and Tamil have resulted in the creation of a prestigious, norm forming variety SSLEand Other Varieties of Sri Lankan Englishes (OVSLEes). Adherence to SSLE phonological norms (Gunesekera, 2005: 121-2) identifies SSLE speech populations. Flouting the norms of SSLE defines the speech populations of OVSLEes. Furthermore several distinctive language specific markedness constraints unique to Tamil influence the branching out of the T/OVSLE from the S/OVSLE bilingual speech communities within the OVSLEes typology. Widyalankara (2014: 113) providing documentary evidence defines and confirmsthat the acoustic deviations in the pronunciation of S/OVSLE and T/OVSLEbilingual speech populations have resulted in the formation of two speech populations. The diversity of these two OVSLEes is more robustly evidenced in pronunciation than in grammar and vocabulary, in the segmental more than the supra segmental. But devoid of this diversity L1 dominancy in the code repertoire of these two bilingual speech populationsinfluence the rate of occurrence of deviations from SSLE pronunciation. The objective of this study is to investigate thecorrelation.

\subsection{Research Question}

\section{Methodology}

Is there a correlation between L1 dominancy in S/OVSLE and T/OVSLE bilinguals and the Rate of occurrence of selected deviations from SSLE pronunciation?

\section{Hypotheses:}

If $\mathrm{X}$ is the estimated L1 dominancy in an S/OVSLE and T/OVSLE bilingual and Y the frequency of occurrence of selected deviations from SSLE in his/her pronunciation:

$\mathbf{H}_{\mathbf{0}}$ - L1 dominancy (X) has nocorrelation with the estimated frequencyof occurrence of deviations from SSLE pronunciation $(\mathrm{Y})$.

$\mathbf{H}_{1}$ - L1 dominancy $(\mathrm{X})$ is positively correlated to the estimated frequencyof occurrence of deviations from SSLE pronunciation $(\mathrm{Y})$.

$\mathbf{H}_{2}$ - L1 dominancy (X) is negatively correlated to the estimated frequencyof occurrence of deviations from SSLE pronunciation $(\mathrm{Y})$.

\subsection{Participants}

\subsubsection{Participant population I: S/SLE bilingual undergraduates}

Out of the total population of 1020 S/SLE bilingual undergraduates (2011/2012) from the Faculties of Humanities and Social Sciences of University of Kelaniya, a population of 200 (mean age 21 years) was selected through standard random sampling procedures as respondents to a questionnaire (Appendix A) anda pronunciation elicitation process. Table 1: Instrument II (§ see 3.4.2.1)was utilized for elicitations. Of the 200 participants 54 did not flout SSLE norms in the selected deviations from SSLE in Instrument II. The latter wereeliminated.From the 146 shortlisted participants who belonged to the S/OVSLEbilingualspeech populations 100 were randomly selected as respondents to the questionnaire.

\subsubsection{Participant population II: T/SLE bilinguals}

100 respondents consisting of T/SLEbilingual Advanced Level students (mean age 17 years) from Sandilipay Hindu College, a rural, mixed school situated roughly eight kilometers from the Jaffna town were respondents to a questionnaire (Appendix B). They underwent thesame pronunciation elicitation processutilizing Table 1: Instrument II(§ see 3.4.2.1).Of the 100 participants 15 did not flout SSLE norms in the selected deviations from SSLE in Instrument II. Thus 85 shortlisted participants who belonged to the $\mathrm{T} /$ OVSLEbilingualspeech populationswere respondents to the questionnaire. 


\subsection{Measuring L1 dominancy}

This study surveyed Birdsong (2006b) and Haji-Hassam (2008) ${ }^{]}$to draw from prior literature a standard procedure to identify the dominant language in the subjects of this study and ascertain the rate of dominancy of one language over the other. The issue of language dominance testing is explored with special methods presently utilized for its determination in current literature. In determining language dominance, literature (Birdsong, 2006b; Haji-Hassam, 2008 and others) generally recognize that at least two variables have to be considered. This paper selects self-perceived language skill level difference quantifying Willingness to Communicate and language selection in functional domains as variables and collates the values to arrive at the measurement for L1 dominancy in each participant of the total $(n=185)$ of S/OVSLE $(n=100)$ andT/OVSLE $(\mathrm{n}=85)$ bilingual speech populations.

\subsubsection{Measurement I: Language selection in functional domains in the bilingual participants}

Language dominance in functional domains according to Grosjean (1997: 165) is usually critical in measuring bilingualism. Grosjean (ibid) and De Houwer (2005) concur that bilinguals normally use their languages in different domains with different people and usually have one stronger and one weaker language. Even with simultaneous exposure to two languages in a multitude of domains bilinguals can develop dominance in one language (Bosch \&Sebastián-Gales 2001: 73).

Two questionnaires: Schematic evaluation of bilingual profiles A and B,were utilized to collect data. Two questions (6 and 7) required the respondents to state the language selection (Sinhala/SLE, Tamil/SLE or both languages) during formal and informal discourse in the identified domains. The difference in the use of L1 and/or L2 in the domains was calculated for each participant as measurement I.

\subsubsection{Measurement II: Self rated average difference of skills in L1 and L2}

In measuring the second variable this study is informed by a similar study by Edmonds \&Kiran (2006). They used the analysed informal self-rating (1-7) average of conversational skills (Speaking and Listening) to categorize their English/Spanish bilingual participants as English dominant, Spanish dominant and balanced bilinguals. The overall difference in proficiency in the 4 skills was used to determine language dominancy. Their findings reveal that some bilingual adults reflect a balance in their language abilities. The balanced group showed no significant difference between the two languages in the average skills statistical evaluation. This study reduplicates the experiment of Edmonds and Kiran (2006) but collects data for informal self-rating along Likert scales for all four skills: Reading, Writing, Speaking and Listening in the two languages Sinhala/Tamil and English for each participant through question 5 of the questionnaire.

The Likert scale ranged from 1- basic proficiency to 5 - maximal/advanced proficiency. Then the skill difference was calculated by obtaining the mean average of the self-declared skill values of each participant along the L1 and L2 scales. The final calculation conflated the measurement of the difference in language selection in functional domains with the value obtained for the difference between the self-rating of skills in L1 and L2 to obtain a value for language dominancy in each participant.

Thus the selection of participants is based on the need to satisfy the ethnolinguistic diversity of this study.

\subsection{Inquiry system I}

\subsection{1 instrument I: The Questionnaires}

This instrument collected data on the following from S/OVSLEand T/OVSLE bilingual participants of this study.

\section{a) Personal information}

Data was collected on age, sex, district of residence and ethnicity from all respondents.

b) The questionnaires (Appendices $\mathrm{A}$ and $\mathrm{B}$ ) obtained qualitative assessments for the following main criteria:

1. Self-declared bilingual status (Q 4)

2. Informal self-assessed ability levels in the four skills in English: Speaking, Writing, Reading and Listening (overall and the four individual skills) (Q 5)

3. Data for language selection in functional domains (Q 6) and (Q 7)

4. Self-assessment of language dominancy (Q 8)

\subsection{Inquiry system II}

\subsubsection{Interviews forpronunciation measurement of respondents}

According to Frankel and Wallen, (1996) interviewing is an important way for a researcher to verify or refute the impressions he or she has gained through observation. They consider interviewing as the most important data collection technique a researcher possesses. Concurrence comes from Cheshire et al (2005) who 
state 'phonological variables show up with high frequencies in sociolinguistic interviews, and can be easily elicited through word lists'.

\subsubsection{Instrument II: Word List for pronunciation elicitation}

This research instrument was compiled from lexicon suggested as representing pronunciation features of OVSLEesobtained from literature. The target phonological feature in each lexicon is relatively easy to perceive and define and could be specified binarily for their variety discrimination load. Furthermore the tokens were evaluated for word frequency for English through the Brown Corpus (Francis and Kucera, 1982) and the selected instrument consists of high frequency words.

\subsubsection{Compilation of lexicon and sources}

25 lexica from surveyed literature compiled the list for pronunciation elicitations. Recorded lemmas which give rise to pronunciation deviations from SSLE in bilinguals who use OVSLEeswere obtained from sources and 5 for each target pronunciation area were randomly shortlisted.

Table 1: Instrument II - Lexical tokens for testing target deviations from SSLE in S/OVSLE and T/OVSLE bilinguals

\begin{tabular}{|l|l|l|l|l|l|l|}
\hline \multirow{2}{*}{$\#$} & \multirow{2}{*}{ Target deviation } & \multicolumn{5}{|c|}{ Lexicon with target phoneme/phonotactic feature } \\
\cline { 3 - 6 } & & \multicolumn{2}{|c|}{1} & \multicolumn{2}{c|}{3} & \multicolumn{1}{c|}{5} \\
\hline 1. & $\mathrm{o} / \mathrm{J}$ & bowl & ball & hole & yoghurt & boat \\
\hline 2. & $\mathrm{f} / \mathrm{p}$ & paddyfield & program & past & profit & airport \\
\hline 3. & $\mathrm{~s} / \mathrm{j}$ & auction & push & sheet & pressure & cousin \\
\hline 4. & $\mathrm{i}+\mathrm{s}$ & station & screen & style & smile & screw \\
\hline 5. & Syllable omission & library & identity & exercise & temporary & government \\
\hline
\end{tabular}

\section{Procedure}

Thedata collectors for participant population I (S/SLE) were staff members of the English Language Teaching Unit (University of Kelaniya). They were graduates who had read English as a subject with post graduate qualifications in Linguistics. Participant populations II (T/SLE) from Sandilipay Hindu College, Jaffna was examined by a team of experienced teachers of English (mean average of teaching experience $=12$ years) headed by Ms.YaminiBaskeran. In all data collecting procedures I instructed and supervised the personnel involved and was a parallel data collector. Each member of the data collecting team had exposure to Linguistics, could transcribe using IPA and was sensitive to pronunciation deviations from SSLE in their respective bilingual populations. Their word list had the target deviation in pronunciation highlighted (as in Table 1).

Each respondent was interviewed by a panel of two data collectors. On arrival at the examining locale the respondents handed over their completed questionnaires and read the 25 word list provided to them. They were required to pronounce each word with maximum clarity. The two data collectors recorded whether the target deviation from SSLE was evidenced in the pronunciation of each word. The perceptive accuracy was dependent on both data collectors perceiving evidence for the target deviation from SSLE in a participant. During shortlisting the respondents who did not deviate from SSLE pronunciation were eliminated from the analysis.

\section{Results and analysis}

$5.1 \%$ rate of occurrence of selected deviations from SSLE pronunciation in bilingual speakers of OVSLE 5.1.1 S/OVSLE bilinguals

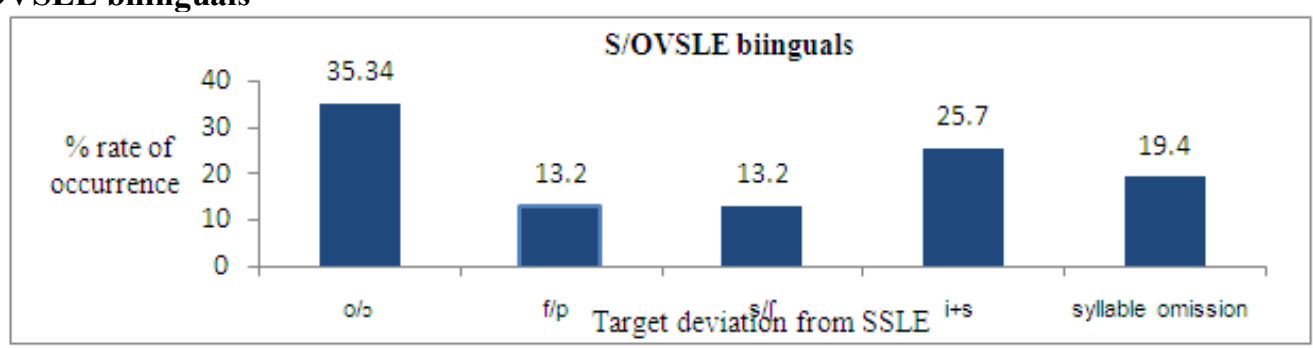

Figure 1: \% rate of occurrence of identified deviations in S/OVSLE bilinguals

The graph indicates that there is a fairly high frequency of occurrence of the deviations from SSLE in the core areas $/ \mathrm{o} / \sim / \mathrm{J} / ; / \mathrm{p} / \sim / \mathrm{f} / ; / \mathrm{s} / \sim \mathrm{J} / ; / \mathrm{s} / \rightarrow /$ is $/$ in S/OVSLE bilingual speakers. 


\subsubsection{T/OVSLE bilinguals}

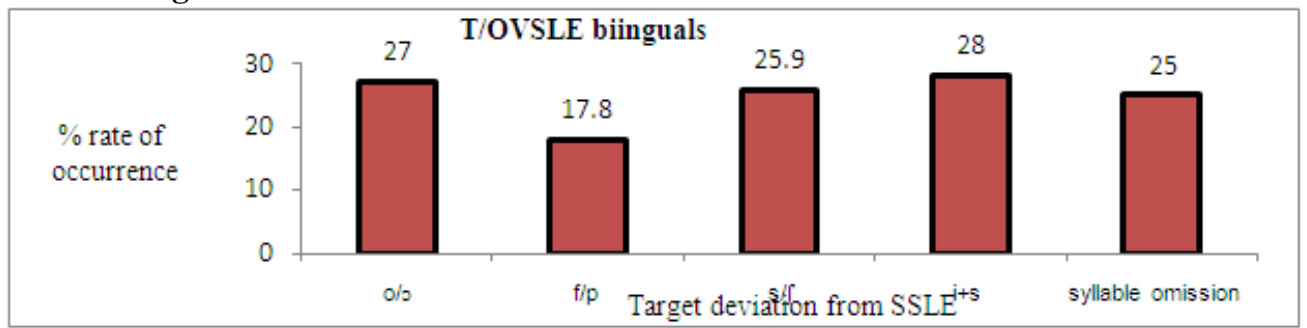

Figure 2: \% rate of occurrence of identified pronunciation deviations from SSLE in T/OVSLE bilinguals

\subsection{Analysis of correlation between L1 Dominancy and Rate of occurrence of deviations 5.2.1 ANOVA for the Research question}

A one-way Analysis of Variance (ANOVA) carried out for L1 dominancywith regard to the two populations of this study suggested that the means of all populations are not equal. Thus with regard to L1 dominancy the null hypothesis is rejected at 5\% level of significance since the p-value obtained $(0.000)$ is less than 0.05 . Therefore, as the means are not equal for the 2 populations, it was decided to analyse them separately with respect to the hypotheses proposed for the research question.

\subsubsection{The Pearson correlation}

The Pearson correlation is used statistically to validate/nullify hypotheses constructed for Research question of this study. The Pearson correlation calculates $p$ value for every data set and records the significance level. According to Rasinger (2008) any p-value less than .05 indicates that the result is not due to chance but due to actual correlation between the variables.

For value of $r$ (the strength of the relationship) interpretation this thesis informed by the following: According to Higgins (2005: 212) for values of $r$ between

- $0.9-1$ : the correlation is very strong.

- $0.7-0.89$ : correlation is strong.

- $0.5-0.69$ : correlation is moderate.

- $0.3-0.4 .9$ : correlation is moderate to low.

- $0.16-0.29$ : correlation is weak to low.

- 0.16 - correlation is too low to be meaningful.

Furthermore for the Pearson Correlation

- 1.0: Perfect positive correlation

- $\quad$-1.0: Perfect negative or inverse correlation.

In the calculation of $\mathrm{R}^{2}$ - the Coefficient of Determination, according to BrownandRodgers (2002: 190) and Gieles (1999) in terms of percentage of variance explained, small is $1 \%$, medium is $9 \%$, and large is $25 \%$.

Based on the above, the hypotheses of the Research question are examined through two analyses: scatter plot graphics and correlational procedures.

\subsubsection{L1 dominancy and rate of occurrence of pronunciation deviations from SSLE in S/OVSLE, and} T/OVSLE bilinguals: Scatter plot graphics

\subsubsection{Scatter plot graphics: S/OVSLE bilinguals}

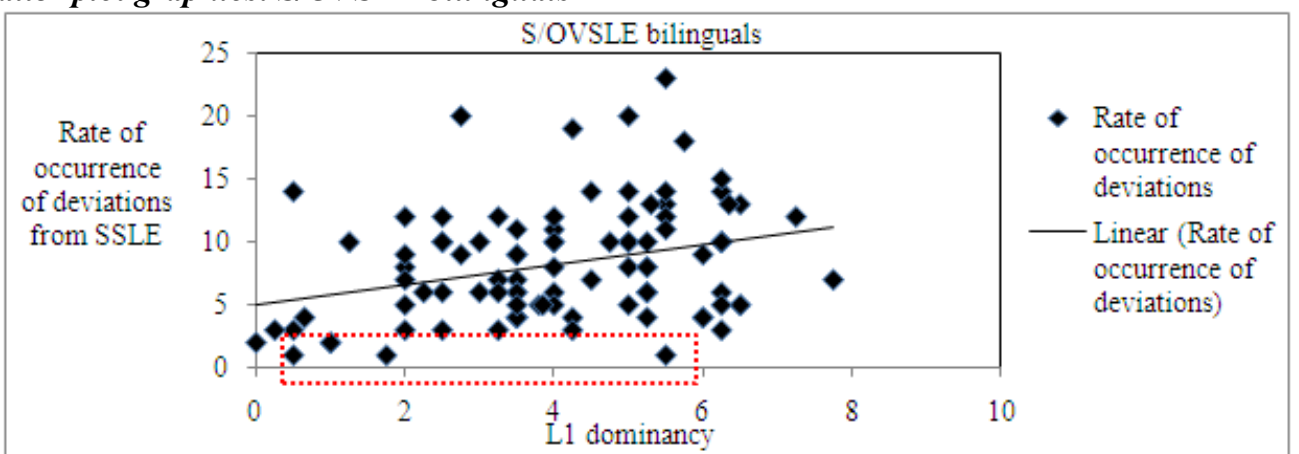

Figure 3: Scatter diagram illustrating correlation between L1 dominancy and rate of occurrence of pronunciation deviations from SSLE in S/OVSLE bilinguals 


\subsubsection{Scatter plot graphics: T/OVSLE bilinguals}

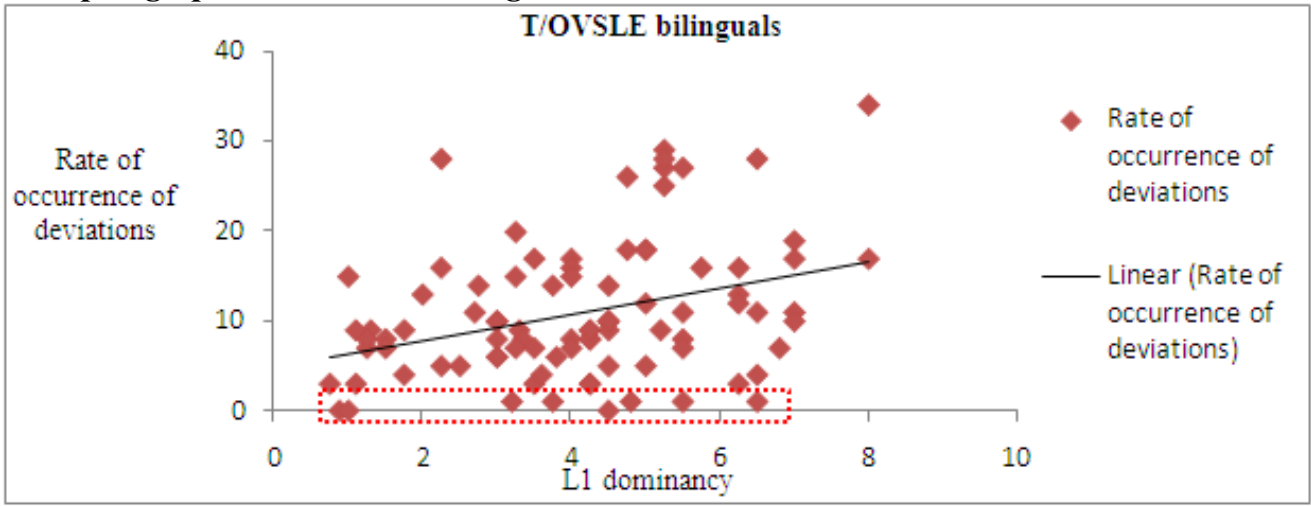

Figure 4: Scatter diagram illustrating correlation between L1 dominancy and rate of occurrence of pronunciation deviations from SSLE in T/OVSLE bilinguals

Data points placed on the $\mathrm{x}$-axis

The dispersion of the data points in the above scatter plots in Figures 3 and 4 illustrate that there is a moderate positive correlation between the two variables in the two populations. Thus the independent variableL1 dominancy affects the dependent variable Rate of occurrence of deviations from SSLE pronunciation in the two populations and indicates a positive relationship.

But note the dispersion of the data points in the above scatter plots indicate that L1 dominancy cannot be the only gauge for the rate of occurrence of deviations from SSLE pronunciation of individual bilinguals in the two populations as many data points disperse away from the linear trendline. Especially note the data points placed on the $\mathrm{x}$-axis of Figures 3 and 4 within the dotted rectangle. The $\%$ rate of occurrence of deviations from SSLE pronunciation of these participants is 0 but they vary in L1 dominancy. This demonstrates that other variables too influence individual rate of occurrence of pronunciation deviations from SSLE.

\subsubsection{Analysis of correlation between L1 Dominancy and Rate of occurrence of deviations \\ 5.2.4.1 Descriptive statistics forS/OVSLE bilingual population}

The correlation between L1 dominancy and the rate of occurrence of deviations for the S/OVSLE bilingual population is 0.309 i.e. the two variables of interest are moderately positively correlated.

Thus for the S/OVSLE bilingual population,

$\mathbf{H}_{1}: \mathrm{L} 1$ is positively correlated to the estimated frequencyof occurrence of deviations from SSLE pronunciation is validated.

Furthermore the correlations are statistically significant at the $5 \%$ level. Any p-values less than .05 indicate that the result is not due to chance. Thus the p-value of 0.002 evidences that there is an actual correlation between the two variables.

$\mathrm{R}^{2}$ or the Coefficient of Determination evaluates the shared variance between the two sets of numbers. Calculation of $\mathrm{R}^{2}$ was conducted through squaring the Pearson Correlation. The correlation is .309. Thus $\mathrm{R}^{2}=$ $.309 \times .309=0.09$. So the variance explained is $0.09 \times 100=0.9 \%$. Thus the coefficient of .309 shows a medium 9\% (BrownandRodgers, 2002: 190) ${ }^{[38]}$ shared variance between the two sets of numbers. This also means that $91.0 \%$ of the variance is unexplained. Thus it could be postulated that factors other than L1 dominancy influences rate of occurrence of deviations from SSLE pronunciation within this population.

\subsubsection{Descriptive statistics for T/OVSLE bilingual population}

The Pearson Correlation between L1 dominancy and Rate of occurrence of deviations is 0.340 i.e. the two variables of interest are moderately positively correlated.

Thus for the T/OVSLE bilingual population,

$\mathbf{H}_{1}:$ L1 dominancy is positively correlated to the estimated frequencyof occurrence of deviations from SSLE pronunciation is validated.

Furthermore the correlations are statistically significant at the 5\% level. Any p-values less than .05 indicate that the result is not due to chance. Thus the p-value of 0.001 evidences that there is an actual correlation between the variables. 
Based on the analysis the correlation between L1 dominancy and rate of occurrence of deviations is 0.340 , i.e. the two variables of interest are slightly positively correlated. Higgins (2005) states that for values of $\mathrm{r}$ between 0.3 and 0.4 .9 , correlation is moderate.

Calculation of $\mathrm{R}^{2}$ was conducted through squaring the Pearson Correlation. The correlation is 0.340 . Thus $\mathrm{R}^{2}=0.340 \times 0.340=0.11$. So the variance explained is $0.11 \times 100=11 \%$. Thus the coefficient of 0.340 shows a medium to large $11 \%$ variance explained. Recall that BrownandRodgers (2002: 190) state medium is $9 \%$, and large is $25 \%$. This also means that $89 \%$ of the variance is unexplained which indicates that influences other than L1 dominancy effect on rate of occurrence of deviations from SSLE in the T/OVSLE bilingual population.

Summation of the descriptive statistics for T/OVSLE bilingual population: There is a positive correlation between the two variables L1 dominancy and the rate of occurrence of deviations from SSLE where $r=0.340 ; p=0.001 ; R^{2}=0.1$ and percentage of variance explained $=11 \%$.

Table 2: Percentage of variance explained for the two populations of this study

\begin{tabular}{|l|c|c|c|}
\hline \multirow{2}{*}{ Variable } & \multicolumn{3}{|c|}{$\%$ of variance explained } \\
\cline { 2 - 4 } & S/OVSLE & T/OVSLE & Average \\
\hline L1 dominancy & 9.0 & 11.0 & 10.0 \\
\hline
\end{tabular}

The $\%$ of variance unexplained in both populations, as large is $25 \%$ and medium is $9 \%$,is moderatly high. Thus it could be postulated that factors other than L1 dominancy influences rate of occurrence of deviations from SSLE pronunciation within the speech populations of this study.A few such factors which were measured but did not undergo analysis are method of learning/acquiring English;if instructed age of commencement the number of years of English instructionand proficiency in English.

\section{Summary Statement}

This study examined the correlation between L1 dominancy and the Rate of occurrence of selected deviations from SSLE pronunciation. The measure for L1 dominancy was a collation of language selection in 15 functional domains and the self-assessed skill difference between L1 and L2 quantifying Willingness To Communicate in a participant population of 185 .

Generalizing the findings above it could be concluded that there is a significant positive correlation between L1 dominancy and Rate of occurrence of selected deviations from SSLE pronunciation in S/OVSLE and T/OVSLE bilinguals. Furthermore responding to question 9 of the questionnaire, 'Out of the languages you speak, which one is dominant?' all participants claimed their mother tongue is dominant. Analyzing language selection in functional domains it was noticed that L1 usage had a significantly higher $\%$ mean than L2 usage in all participants.

Thus in conclusion it could be stated that in the OVSLE speech populations the self-assessment that you are stronger in L1 skills resulted in more use of L1 in discourse domains.Additionally self- assessed proficiency quantifies the Willingness To Communicate in a language (Dewaele, 2010; Trofimovich, 2011) and reflects how one perceives one's competence is in the L2is likely to be and captures the implications of psychological traits such as communication apprehension, introversion, reticence, and shyness on L2 communicative behavior.

The corollary is that as Willingness To Communicate in L2 decreases L1 dominancy in discourse increases. Senarathne (2009: 55) discussing English loanword phonology in Sinhala states that 'in lone lexical items occurring in predominant Sinhala utterances, these nativizations are not categorized as mistakes or errors'. Thus the increase in L1 dominancy in functional domains results in a high exposure to/usage of loanword phonology which violates SSLE norms and literature along with the findings of this study bear proof forOVSLE speech populations transferring the loan phonology to L2 speech discourse. This in turn increases the rate of occurrence of deviations from SSLE in English discourse. Furthermore as L1 usage especially in functional domains increases, the exposure to SSLE pronunciation decreases in users ofOVSLEes. The lack of exposure to SSLE pronunciation too results in the inability in OVSLE bilinguals to self-comprehend that they are deviating from SSLE pronunciation. For example of a 185 participant OVSLE speech population an average of $81 \%$ watched only Sinhala/Tamil teledramas and films. $86 \%$ accessed vernacular news bulletins and listened to Sinhala/Tamil music. Furthermore in SMSs the language is Sinhala/Tamil though the font is English. Moreover what was noted is that graphemic representations of loanwords in the matrix Sinhala/Tamil sentences in Sinhala/Tamil print media/publications very often reflected deviations from SSLE. In electronic media too the advent of Colloquial Spoken Sinhala/Tamil gives currency to assimilated loanword phonology and influences the pronunciation of OVSLE bilinguals.

In sum the bias towards L1 during language selection in functional domains and the lack of Willingness To Communicate in L2 results in L1 dominancy in bilinguals. As a consequence the greater exposure to and a higher use of loanword phonology in L1 discourse transfers to L2. This increases the rate of 
occurrence of deviations from SSLE norms in OVSLE pronunciation. Thus L1 dominancy reflected through self-rated language skills and language selection in functional domains is a causal factor for dialectal variation in SLE pronunciation.

\section{References}

[1]. J.Sia and J.M. Dewaele, Are you bilingual? Birkbeck Studies in Applied Linguistics.1, 2006, 1 - 19.

[2]. L. Bloomfield, Language. George Allen \&Unwin: London, 1935.

[3]. F. Grosjean, The bilingual individual,Interpreting. 2, 1997,163-187.

[4]. K. Hakuta, Mirror of language: The debate on bilingualism. New York: Basic, 1986.

[5]. E. Haugen, The Norwegian language in America: A study in bilingual behavior. Filadelfia: University of Pennsylvania Press, 1953.

[6]. H. B. Beardsmore, Bilingualism: Basic Principles. Multilingual Matters, 1982.

[7]. W. F. Mackey, The Description of Bilingualism, Journal of the Canadian Linguistic Association, 1957.

[8]. S. Arsenian, Bilingualism and mental development, New York: Columbia University Press, 1937.

[9]. D. Crystal, The Cambridge Encylopedia of the English Language, 2nd edition. Cambridge University Press, 2003.

[10]. J.Abutalebi and D. Green, Bilingual language production: The neurocognition of language representation and control. Journal of Neurolinguistics. 20,2007, 242-275.

[11]. A. M. B. de Groot,Language and Cognition in Bilinguals and Multilinguals: An Introduction. Psychology Press, 2010.

[12]. M.Finkbeiner, T. H. Gollan and A. Caramazza, Lexical access in bilingual speakers: What's the (hard)problem? Bilingualism: Language and Cognition. 9 (2), 2006,153-166.

[13]. W. S. Francis, Cognitive integration of language and memory in bilinguals: semantic representation. Psychological Bulletin.125, $1999,193-222$.

[14]. J. F. Kroll and E. Stewart, Category interference in translation and picture naming: Evidence for asymmetric connections between bilingual memory representations. Journal of Memory and Language. 33, 1994, 149-174.

[15]. M. Gunesekera, ThePost Colonial Identity of Sri Lankan English.Katha Publishers, Colombo,2005.

[16]. R. C. Widyalankara, Dialectal variation in Sri Lankan English Pronunciation: An acoustic analysis. LAP Publications: Amazon, 2014, ISBN- 978-3-8484-3484-8.

[17]. D. Birdsong, Dominance, proficiency, and second language grammatical processing. Applied Psycholinguistics.27,2006b, 46-49.

[18]. I. A. Haji-Hassam, A bilingual s linguistic skills and language dominance: adescriptive study. SosyalBilimlerEnstitüsüDergisi Say. 25(1),2008, 89-106. (Retrieved on May 12, 2012). http://sbe.erciyes.edu.tr/dergi/sayi 25/7-\%20 89-106.\%20syf. .pdf

[19]. F. Grosjean, The bilingual individual. Interpreting. 2, 1997,163-187.

[20]. A. De Houwer, Early bilingual acquisition: Focus on morphosyntax and the separate development hypothesis. In J. Kroll., A. De Groot (eds). The handbook of bilingualism, 2005, 30-48. Oxford, UK: Oxford University Press.

[21]. L.Bosch andN.Sebastian-Galles, Early language discrimination capacities in infants from bilingual environments. In A. M. Barrena., A. Ezeizabarrena., M. J. Idiazabal., I. MacWhinney., B. (eds). Research on Child Language Acquisition. 1(2), 2001, 95-103.

[22]. L. A.Edmonds and S. Kiran,Effect of semantic naming treatment on crosslinguistic generalization in bilingual aphasia. Journal of Speech, Language, and Hearing Research.49, 2006, 729-748.

[23]. J. R. Fraenkel and N. E. Wallen, How to design and evaluate research in education (3rd ed.). New York: McGraw-Hill, 1996.

[24]. J. Cheshire, P.Kerswill and A. WilliamsOn the non-convergence of phonology, grammar and discourse, 2005.(Retrieved on March 15, 2013).http://webspace.qmul.ac.uk/jlcheshire/pdf $\% 20$ papers $/$ chesh $\% 20 \mathrm{kers} \% 20 \% 26 \% 20 \mathrm{williams} 3$.pdf

[25]. W. Francis andH. Kučera, Frequency analysis of English usage: Lexicon and grammar. Boston: Houghton Mifflin, 1982.

[26]. S. M. Rasinger, Quantitative Research in Linguistics: An Introduction. Continuum International Publishing Group, 2008.

[27]. E. T.Higgins, Value from regulatory fit. Current Directions in Psychological Science. 14, 2005, 209-213.

[28]. J. D.Brown and T. Rodgers,Doing Second Language Research. Oxford: Oxford University Press, 2002.

[29]. F. Gieles, An explanation of the statistics used in the Meta-analysis, 1999. (Retrieved on April 20, 2012).http://www.humanbeing.demon.nl/humanbeingsweb/Library/explanation.htm

[30]. J. Dewaele,Multilingualism and affordances: Variation in self-perceived communicative competence and communicative anxiety in French L1, L2, L3 and L4.IRAL. 48, 2010, 105-129.

[31]. P. Trofimovich, Language experience in L2 phonological learning: Effects of Psycholinguistic and sociolinguistic variables. InternationalReviewof Applied Linguistics. 49, 2011, 135-156.

[32]. C. D. Senarathne, Sinhala- English code mixing in Sri Lanka: A sociolinguistic study. LOT: Netherlands, 2009.

\section{Appendix A: Schematic evaluation of bilingual profiles: Sinhala/Sri Lankan English undergraduates}

Name ....

Student \#.

Age $\ldots . . . . . . . . . . .$.

Sex: Male/Female

1. What was your performance level in the followingexaminations? Circle the grade obtained if applicable.

\begin{tabular}{|l|c|l|l|l|l|}
\hline Examination & \multicolumn{5}{|c|}{ Grade obtained } \\
\hline O/L Sinhala & A & B & C & S & W \\
\hline O/L Tamil & A & B & C & S & W \\
\hline A/L Sinhala & A & B & C & S & W \\
\hline O/L English Language & A & B & C & S & W \\
\hline O/L English Literature & A & B & C & S & W \\
\hline A/L General English & A & B & C & S & W \\
\hline
\end{tabular}




\section{The appropriate box in the following}

2. What was your method of learning English?

\begin{tabular}{|l|l|}
\hline Instructed in school & \\
\hline Natural acquisition at home & \\
\hline Both & \\
\hline
\end{tabular}

3. If instructed \# of years of English instruction in school/s:

\begin{tabular}{|l|l|l|l|l|}
\hline \multirow{3}{*}{ Grade } & $1-13$ & $1-11$ & $3-11$ & $3-13$ \\
\cline { 2 - 5 } & & & & \\
\hline
\end{tabular}

4. Are you a bilingual in Sinhala /Sri Lankan English?

\begin{tabular}{|l|l|}
\hline Yes & No \\
\hline & \\
\hline
\end{tabular}

5. If bilingual what is your overall proficiency and skills in the languages along a scale of $1-5$ ?

\section{The appropriate box in the following}

(a) Overall Proficiency in Sinhala

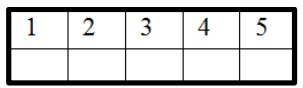

(b) Skills in Sinhala

Speaking

Writing
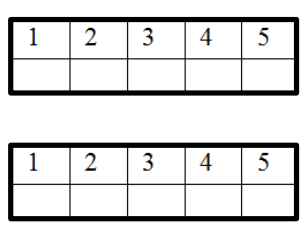

Reading

Listening
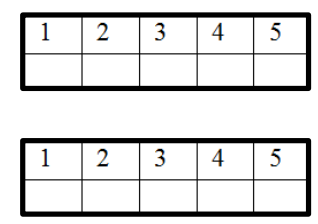

Overall proficiency in English

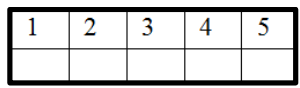

Skills in English
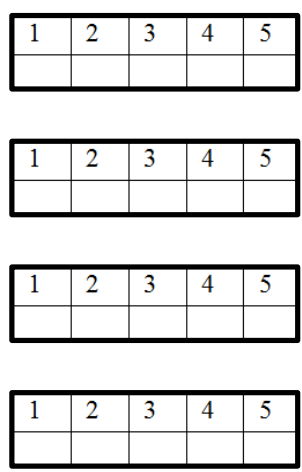

Write $S$ if Sinhala, $E$ if English, $S$ and $E$ if you use both languages.

$\boldsymbol{F}$-formal, $\mathbf{I}$ - informal, $\boldsymbol{A}$ - academic, $\boldsymbol{N}$ - novels

6. What language(s) do you use for/with .......

\begin{tabular}{|c|l|l|l|l|l|l|l|c|}
\hline \multicolumn{2}{|c|}{ Letters } & Parents & Siblings & Friends & Priests & Lecturers & $\begin{array}{c}\text { Administrative } \\
\text { staff }\end{array}$ & $\begin{array}{c}\text { Dean } \\
\text { /VC }\end{array}$ \\
\cline { 1 - 4 } & & & & & & & \\
\hline
\end{tabular}

7. What language(s) do you use when you listen/read/watch/ write .......

\begin{tabular}{|c|c|c|c|c|c|c|c|c|}
\hline \multirow[t]{2}{*}{ Radio } & \multirow[t]{2}{*}{ Internet } & \multirow[t]{2}{*}{ Newspapers } & \multicolumn{2}{|c|}{ Books } & \multirow{2}{*}{$\begin{array}{l}\text { Films/ news/ } \\
\text { teledramas }\end{array}$} & \multirow[t]{2}{*}{ Music } & \multirow[t]{2}{*}{ SMSs } & \multirow[t]{2}{*}{ Tutorials } \\
\hline & & & A & $\mathrm{N}$ & & & & \\
\hline & & & & & & & & \\
\hline
\end{tabular}


8. Out of the two languages Sinhala and Sri Lankan English, which one is dominant?

$\checkmark \quad$ Tick your answer.

\begin{tabular}{|l|l|}
\hline Sinhala & Sri Lankan English \\
\hline & \\
\hline
\end{tabular}

\section{Answer the following question. Use Sinhala if necessary.}

9. Why do you consider the selected language to be dominant?

10. Now

- Please handover this form to the data collector

- $\quad$ Then you are required to read out loud a selection of English words.

- $\quad$ This is needed purely for experimental purposes.

- $\quad$ Start pronouncing the words given in a list to you at the signal to commence.

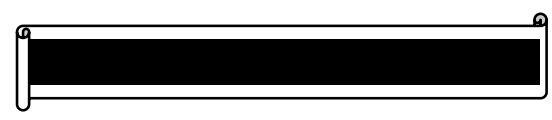

\section{Official use only}

Data collector's assessment of SLE pronunciation in Sinhala/SLE bilinguals

$\checkmark \quad$ Indicates the correct pronunciation. An X mark denotes that the target deviation is present. Please insert any other deviations in the third column.

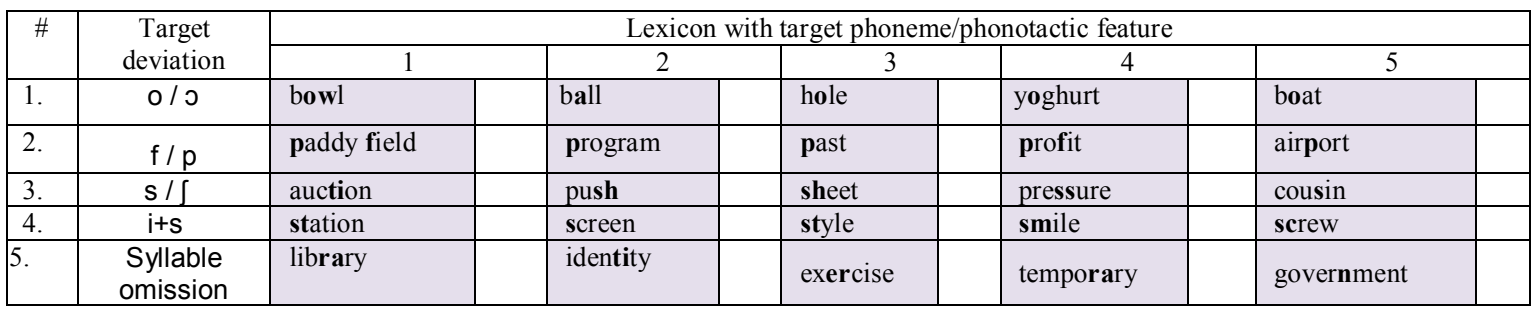

Appendix B: Schematic evaluation of bilingual profiles: Tamil/Sri Lankan English students Name

Age

Sex: Male/Female

1. What was your performance level in the following examinations? Circle the grade obtained if applicable.

\begin{tabular}{|l|c|c|c|c|c|}
\hline Examination & \multicolumn{5}{|c|}{ Grade obtained } \\
\hline O/L Tamil & A & B & C & S & W \\
\hline O/L Sinhala & A & B & C & S & W \\
\hline O/L English Language & A & B & C & S & W \\
\hline O/L English Literature & A & B & C & S & W \\
\hline
\end{tabular}

2. What was your method of learning English?

\begin{tabular}{|l|l|}
\hline Instructed in school & \\
\hline Natural acquisition at home & \\
\hline Both & \\
\hline
\end{tabular}

3. If instructed \# of years of English instruction in school/s: 


\begin{tabular}{|l|l|l|l|l|}
\hline Grade & $1-13$ & $1-11$ & $3-11$ & $3-13$ \\
\cline { 2 - 5 } & & & & \\
\hline
\end{tabular}

4. Are you a bilingual in Sinhala /Sri Lankan English?

\begin{tabular}{|l|l|}
\hline Yes & No \\
\hline & \\
\hline
\end{tabular}

5. If bilingual what is your overall proficiency and skills in the languages along a scale of $1-5$ ?

$\checkmark$ The appropriate box in the following

(a) Overall Proficiency in Sinhala

\begin{tabular}{|l|l|l|l|l|}
\hline 1 & 2 & 3 & 4 & 5 \\
\hline & & & & \\
\hline
\end{tabular}

(b)

Speaking

Skills in Sinhala

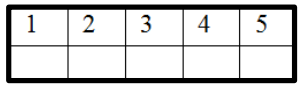

Writing

Reading
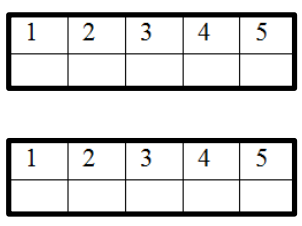

Listening

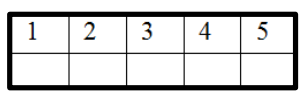

Overall proficiency in English

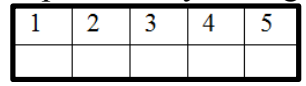

Skills in English
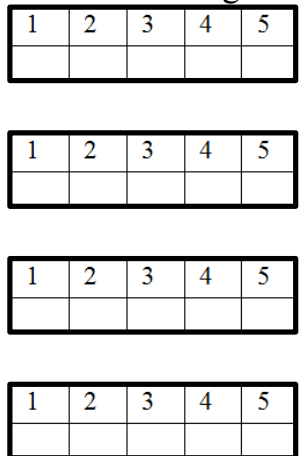

Write $T$ if Tamil, $E$ if English, $T$ and $E$ if you use both languages in the box.

$\boldsymbol{F}$-formal, I- informal, $\boldsymbol{A}$ - academic, $\boldsymbol{N}$ - novels

6. What language(s) do you use with .......

\begin{tabular}{|c|c|c|c|c|c|c|}
\hline Parents & Siblings & Friends & Priests & Teachers & Office staff & The principal \\
\hline & & & & & & \\
\hline
\end{tabular}

7. What language(s) do you use when you listen $/ \mathrm{read} / \mathrm{watch} /$ write .......

\begin{tabular}{|l|l|l|l|l|l|l|l|l|l|}
\hline Radio & Internet & \multirow{2}{*}{ Newspapers } & \multicolumn{2}{|c|}{ Books } & Films/news/teledramas & Music & \multicolumn{2}{|l|}{ SMSs } & Letters \\
\cline { 7 - 9 } & & & & & & & F & I \\
\hline
\end{tabular}

8. Out of the languages you speak, which one is dominant?

\begin{tabular}{|l|l|}
\hline Tamil & Sri Lankan English \\
\hline & \\
\hline
\end{tabular}

Answer the following question. Use Sinhala if necessary.

9. Why do you consider the selected language to be dominant?

10.

- Please handover this form to the data collector

- Now you are required to read out loud a selection of English words.

- This is needed purely for experimental purposes.

- Start pronouncing the words given in a list to you at the signal to commence. 


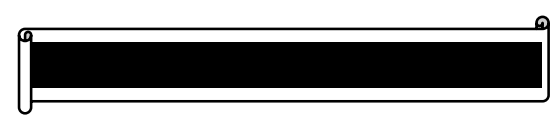

Official use only

Data collector's assessment of SLE pronunciation in Sinhala/SLE bilinguals

$\checkmark \quad$ Indicates the correct pronunciation. An $\boldsymbol{X}$ mark denotes that the target deviation is present. Please insert any other deviations in the third column.

\begin{tabular}{|c|c|c|c|c|c|c|}
\hline \multirow[t]{2}{*}{ \# } & \multirow{2}{*}{$\begin{array}{c}\text { Target } \\
\text { deviation }\end{array}$} & \multicolumn{5}{|c|}{ Lexicon with target phoneme/phonotactic feature } \\
\hline & & 1 & 2 & 3 & 4 & 5 \\
\hline 1. & $0 / 0$ & bowl & ball & hole & yoghurt & boat \\
\hline 2. & $f / p$ & $\begin{array}{l}\text { paddy } \\
\text { field }\end{array}$ & program & past & profit & airport \\
\hline 3. & $\mathrm{~s} / \mathrm{s}$ & auction & push & sheet & pressure & cousin \\
\hline 4. & $\mathrm{i}+\mathrm{s}$ & station & screen & style & smile & screw \\
\hline 5. & $\begin{array}{l}\text { Syllable } \\
\text { omission }\end{array}$ & library & identity & exercise & temporary & government \\
\hline
\end{tabular}

